\title{
APOA5 -1131T/C polymorphism and coronary artery disease susceptibility in Chinese population: an updated meta-analysis and review
}

\author{
J. Zhang, D.G. Wan, H.L. Song and W.G. Zhang \\ The Second Affiliated Hospital of Zhengzhou University, Zhengzhou, China \\ Corresponding author: W.G. Zhang \\ E-mail: zhangwg71@126.com \\ Genet. Mol. Res. 14 (4): 12330-12339 (2015) \\ Received May 12, 2015 \\ Accepted August 19, 2015 \\ Published October 9, 2015 \\ DOI http://dx.doi.org/10.4238/2015.October.9.22
}

\begin{abstract}
Although many studies have investigated the association of the APOA5 - $1131 \mathrm{~T} / \mathrm{C}$ polymorphism with coronary artery disease (CAD), definite conclusions have not been drawn. To understand the effects of the APOA5 -1131T/C polymorphism on the risk of developing CAD, we performed an updated meta-analysis in the Chinese population. Relevant studies published till April 2015 were identified from databases such as PubMed, Springer Link, Ovid, Chinese Wanfang Data Knowledge Service Platform, Chinese National Knowledge Infrastructure, and Chinese Biology Medicine. A total of 19 studies including 3983 patients and 4358 controls were involved in this meta-analysis. The crude OR with $95 \% \mathrm{Cl}$ was calculated to assess the strength of the association. With the pooled data from the studies included in this meta-analysis, we found a significant association between the APOA5 -1131T/C polymorphism and CAD risk in the Chinese population (C vs T: OR $=1.34,95 \% \mathrm{Cl}=1.16-1.54 ; \mathrm{CC}$ vs TT: $\mathrm{OR}=1.73,95 \% \mathrm{Cl}=1.30-2.30 ; \mathrm{CC}$ vs TT and TC: $\mathrm{OR}=1.51,95 \% \mathrm{Cl}=$ 1.17-1.95; $\mathrm{CC}$ vs TC: $\mathrm{OR}=1.30,95 \% \mathrm{Cl}=1.03-1.65)$. Stratified analyses
\end{abstract}


according to the geographical location and source of controls revealed significantly increased risk in South China and in population-based studies. In conclusion, our meta-analysis provides substantial evidence that the APOA5 -1131T/C polymorphism might contribute to CAD development in the Chinese population.

Key words: Meta-analysis; APOA5 -1131T/C; Coronary artery disease; Polymorphism

\section{INTRODUCTION}

Cardiovascular disease is responsible for $30 \%$ of all deaths worldwide, with most of its current burden being in developing countries (Gaziano, 2005). After a peak around 1968, death from coronary artery disease (CAD) has declined significantly in the United States (Rosamond et al., 2007). However, in China, CAD has become a public health problem in the past few decades. The overall CAD mortality rate (per 100,000 individuals per year) in China rose from 95.3 in 1999 to 103.4 in 2008 (Jiang et al., 2012). In 2008, the crude morbidity rate of ischemic heart disease was $12.7 \%$ among Chinese urban residents (Li et al., 2013a). Epidemiological studies have identified several risk factors for CAD including age, gender, hypertension, diabetes mellitus, hypercholesterolemia, smoking, and family history (Dalen et al., 2014). However, only a subset of individuals exposed to these risk factors eventually develop CAD indicating a pivotal role of genetic factors in susceptibility to CAD. Most recently, genomic studies have revealed a series of new candidate markers that may contribute to the pathogenesis of CAD (Sayols-Baixeras et al., 2014). Among these, apolipoprotein A5 (APOA5), a relatively new member of the apolipoprotein family discovered in 2001 is of significance (Pennacchio et al., 2001; van der Vliet et al., 2001). Evidence suggests that APOA5 plays a crucial role in triglycerides metabolism (van Dijk et al., 2004) and increased level of plasma triglycerides is known to predict the prevalence of CAD (Hokason et al., 1996).

Since the discovery of the APOA5 gene and its association with triglycerides, 16 single nucleotide polymorphisms (SNPs) have been discovered in this gene (Li et al., 2013b). Among these SNPs, -1131T/C gene polymorphism was considered to be closely associated with CAD morbidity (Zhang et al., 2011). The first report on the association between APOA5 -1131T/C polymorphism and CAD was by Szalai and co-workers among the Hungarian population (Szalai et al., 2004). Although many subsequent studies analyzed the influence of APOA5 -1131T/C polymorphism on CAD risk, no clear consensus has been reached. Two meta-analyses (Zhai et al., 2011; Li et al., $2013 b)$ have been conducted to investigate the relationship between APOA5 -1131T/C and CAD in Chinese population. However, there is scope for improvement in these studies. Therefore, we have performed this updated meta-analysis to understand the relationship of APOA5 -1131T/C polymorphism with risk of CAD in Chinese population. In addition, we have implemented subgroup analyses stratified by geographic location and the source of control population to explore the possible effects of gene-environment interactions with respect to CAD risk.

\section{MATERIAL AND METHODS}

\section{Literature search}

A comprehensive literature search was performed using PubMed, Springer Link, 
Ovid, Chinese Wanfang Data Knowledge Service Platform, Chinese National Knowledge Infrastructure, and Chinese Biology Medicine for relevant published articles with the following MeSH terms: ("APOA5" OR "apolipoprotein A5") AND ("genetic polymorphism" OR "gene" OR "polymorphism") AND ("coronary artery disease" OR "CAD”) AND ("China” OR "Chinese" OR "Taiwan"). An upper date limit of April 6, 2015, was applied but no lower date limit was used. The search was not limited by language restrictions and focused on studies conducted in humans. Concurrently, the reference lists of reviews and retrieved articles were searched manually.

\section{Inclusion/exclusion criteria}

Studies were included if they met the following criteria: 1) case-control study or cohort study on the association between APOA5 -1131T/C polymorphism and CAD risk; 2) patient diagnoses based on angiographic features, clinical or laboratory findings; 3) sufficient published data on sample size, OR, and their $95 \% \mathrm{Cl}$; 4) only Chinese participants; and 5) detailed published information on genotype frequency. Studies were excluded if they were: 1) not case-control or cohort studies, 2) duplicates of a previous publication; 3) based on incomplete data; 4) metaanalyses, letters, reviews, or editorial articles.

\section{Data extraction}

Two independent investigators carefully extracted information from all eligible studies according to the inclusion criteria listed above. The title and abstract of all potential articles were screened to determine their relevance. Full articles were also scrutinized if the title and abstract were ambiguous. The following data was collected from each study: first author's surname, year of publication, geographical location, source of controls, sample size, and the number of subjects with APOA5 -1131T/C genotypes. Disagreements were resolved through discussions between the two authors and if consensus was not achieved, the decision was made by all the authors.

\section{Statistical analysis}

STATA statistical package (version 10, STATA, College Station, TX, USA) was used for statistical analyses. Chi-square $\left(X^{2}\right)$ test was used for Hardy-Weinberg equilibrium of genotypes in the control group of each reviewed study and the heterogeneity of rare allele frequencies in the control groups among all studies. Crude ORs with $95 \% \mathrm{Cls}$ were used to assess the strength of the association between APOA5 -1131T/C polymorphism and CAD risk. Depending on the results of the heterogeneity test of individual studies, the fixed-effect model (Mantel-Haenszel) or the random-effect model (DerSimonian and Laird) was selected to summarize the combined ORs and their $95 \% \mathrm{Cls}$. Sensitivity analysis was conducted using both the models to verify the stability of the meta-analysis. Begg's funnel plot and the Egger linear regression test were used to assess the publication bias. In addition to comparison among all subjects, stratification analyses by geographical location and source of controls was performed. All the $\mathrm{P}$ values were two-sided; $\mathrm{P}$ $<0.05$ was considered to be statistically significant. 


\section{RESULTS}

\section{Eligible studies}

A total of 98 articles regarding APOA5 gene polymorphism with respect to CAD were identified. After screening the titles and abstracts, 72 articles were excluded because they were on non-Chinese population, review articles, duplicates, or irrelevant to the current study. Of the 26 potentially relevant articles, 25 were identified for full study retrieval (Wang, 2004; Bi et al., 2004, 2005; Liu et al., 2005; Tang et al., 2005; Yan et al., 2005; Hsu et al., 2006; Tang, 2006; Yin, 2006; Cheng et al., 2007; Li et al., 2007; Qiu, 2007; Qiu et al., 2007; Yang et al., 2007; Yu et al., 2007; Zhang, 2007; Zhu et al., 2007; Xu and He, 2008; Zhao, 2008; Zhang, 2009; Han et al., 2010; Chen et al., 2011; Han, 2011; Yuan et al., 2011; Dai et al., 2013), one was excluded because it had no controls (Qiu et al., 2007), and five (Wang, 2004; Bi et al., 2005; Tang, 2006; Cheng et al., 2007; Han, 2011) were excluded because they concerned subjects included in an expanded series. Finally, 19 studies (Bi et al., 2004; Liu et al., 2005; Tang et al., 2005; Yan et al., 2005; Yin, 2006; Hsu et al., 2006; Qiu, 2007; Yang et al., 2007; Yu et al., 2007; Zhang, 2007; Zhu et al., 2007; Li et al., 2007; Xu and He, 2008; Zhao, 2008; Zhang, 2009; Han et al., 2010; Chen et al., 2011; Yuan et al., 2011; Dai et al., 2013) including 3983 CAD patients and 4358 controls were involved in this meta-analysis according to the inclusion criteria. The publication year of studies ranged from 2004 to 2013. Fourteen of these studies were written in Chinese and five in English. Figure 1 illustrates the trial flow chart and the characteristics of the included studies are summarized in Table 1.

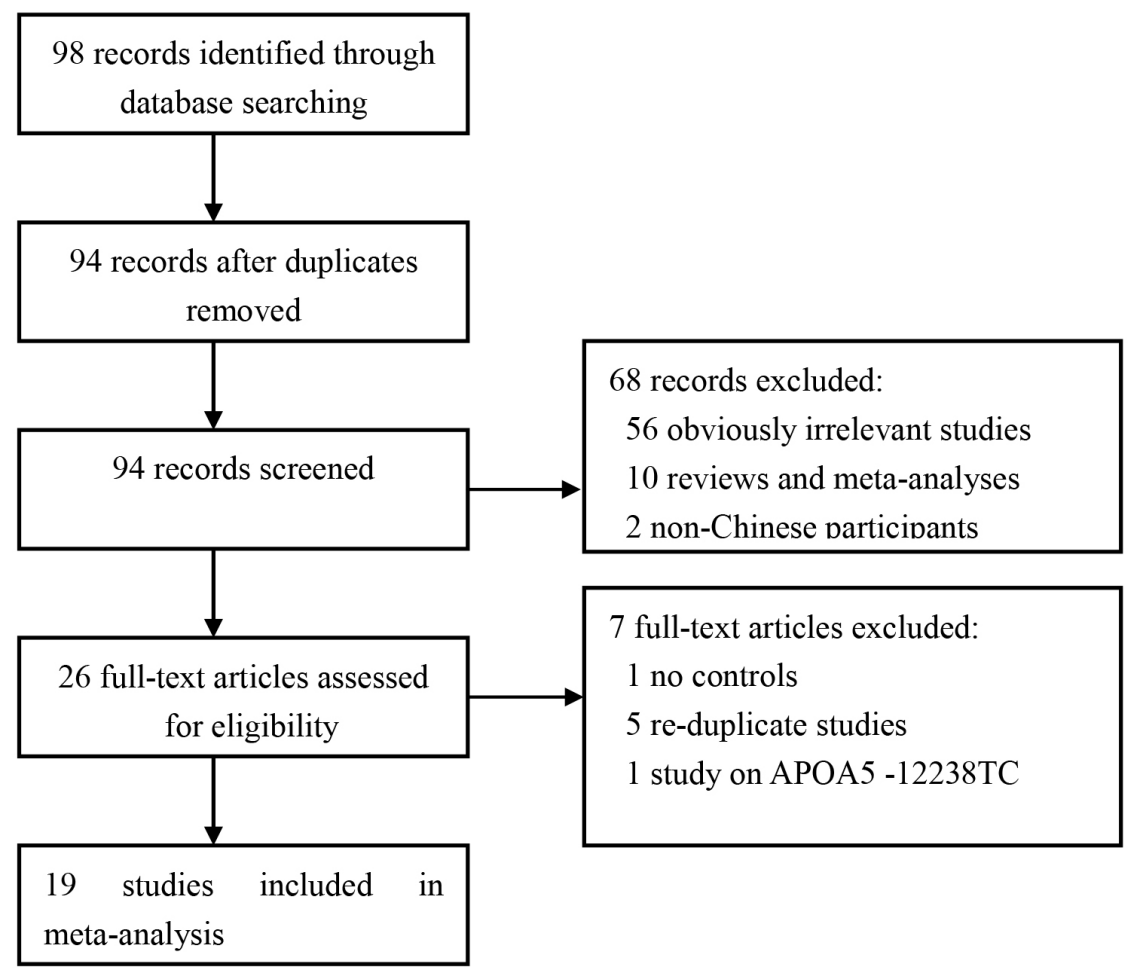

Figure 1. Flow diagram of literature search and selection of eligible studies. 


\begin{tabular}{|c|c|c|c|c|c|c|c|c|c|c|c|c|}
\hline \multirow[t]{2}{*}{ References } & \multirow[t]{2}{*}{ Source of controls } & \multirow[t]{2}{*}{ Geographic location } & \multirow[t]{2}{*}{ No. of cases } & \multirow[t]{2}{*}{ No. of controls } & \multicolumn{3}{|c|}{ Patient } & \multicolumn{3}{|c|}{ Control } & \multicolumn{2}{|c|}{ HWE } \\
\hline & & & & & $\mathrm{TT}$ & $\mathrm{CT}$ & $\mathrm{CC}$ & $\mathrm{TT}$ & $\mathrm{CT}$ & $\mathrm{CC}$ & $x^{2}$ & $P$ \\
\hline Bi 2004 & PB & Beijing & 312 & 317 & 108 & 159 & 45 & 136 & 151 & 30 & 1.67 & 0.196 \\
\hline Tang 2005 & PB & Jiangsu & 235 & 262 & 80 & 120 & 35 & 107 & 130 & 25 & 2.63 & 0.105 \\
\hline Liu 2005 & PB & Sichuan & 483 & 502 & 181 & 226 & 76 & 246 & 212 & 44 & 0.03 & 0.861 \\
\hline Yan 2005 & PB & Beijing & 113 & 155 & 41 & 60 & 12 & 83 & 58 & 14 & 0.69 & 0.407 \\
\hline Yin 2006 & PB & Shandong & 195 & 181 & 71 & 103 & 21 & 78 & 85 & 18 & 0.55 & 0.458 \\
\hline Hsu 2006 & PB & Taiwan & 211 & 317 & 104 & 83 & 24 & 145 & 156 & 16 & 10.22 & 0.001 \\
\hline Qiu 2007 & PB & Jiangsu & 260 & 316 & 126 & 106 & 28 & 177 & 123 & 16 & 0.84 & 0.361 \\
\hline Yang 2007 & $\mathrm{HB}$ & Jiangsu & 168 & 160 & 58 & 80 & 30 & 77 & 67 & 16 & 0.06 & 0.800 \\
\hline Yu 2007 & PB & Beijing & 140 & 156 & 46 & 67 & 27 & 67 & 75 & 14 & 1.18 & 0.277 \\
\hline Zhang 2007 & $\mathrm{HB}$ & Zhejiang & 141 & 129 & 54 & 59 & 28 & 61 & 52 & 16 & 0.87 & 0.351 \\
\hline Zhu 2007 & PB & Hubei & 119 & 210 & 42 & 57 & 20 & 106 & 72 & 32 & 9.89 & 0.002 \\
\hline Li 2007 & PB & Hunan & 186 & 268 & 83 & 49 & 54 & 149 & 66 & 53 & 50.70 & 0.000 \\
\hline Xu 2008 & PB & Shandong & 195 & 181 & 71 & 103 & 21 & 78 & 85 & 18 & 0.55 & 0.458 \\
\hline Zhao 2008 & $\mathrm{HB}$ & Xinjiang & 155 & 145 & 46 & 86 & 23 & 59 & 75 & 11 & 3.80 & 0.051 \\
\hline Zhang 2009 & PB & Xinjiang & 112 & 136 & 54 & 49 & 9 & 95 & 36 & 5 & 0.46 & 0.498 \\
\hline Han 2010 & PB & Shandong & 195 & 181 & 71 & 103 & 21 & 78 & 85 & 18 & 0.55 & 0.458 \\
\hline Chen 2011 & PB & Shanxi & 249 & 176 & 97 & 119 & 33 & 63 & 78 & 35 & 1.45 & 0.229 \\
\hline Yuan 2011 & $\mathrm{HB}$ & Xinjiang & 344 & 408 & 172 & 149 & 23 & 163 & 184 & 61 & 0.59 & 0.444 \\
\hline Dai 2013 & PB & Hunan & 170 & 158 & 74 & 44 & 52 & 101 & 42 & 15 & 9.45 & 0.002 \\
\hline
\end{tabular}

PB: Population-based, HB: hospital-based; HWE: Hardy-Weinberg equilibrium.

\section{Meta-analysis results}

Table 2 lists the primary results. Overall, a significantly elevated risk of CAD was associated with APOA5 $-1131 \mathrm{~T} / \mathrm{C}$ variants (CC vs TT: OR $=1.73,95 \% \mathrm{Cl}=1.30-2.30$; $\mathrm{CC}$ vs TT and TC: $\mathrm{OR}=1.51,95 \% \mathrm{Cl}=1.17-1.95 ; \mathrm{CC}$ vs TC: $\mathrm{OR}=1.30,95 \% \mathrm{Cl}=1.03-1.65)$. For allele $\mathrm{C}$ versus allele $\mathrm{T}$, the pooled $\mathrm{OR}$ was $1.34(95 \% \mathrm{Cl}=1.16-1.54 ; \mathrm{P}=0.000$ for heterogeneity) (Figure 2). However, there was significant heterogeneity between studies. Hence, subgroup analyses by geographical location and source of controls was performed. In the stratified analysis by geographical location, significantly increased risk was found in the population from South China (C vs T: OR $=1.48,95 \% \mathrm{Cl}=1.29-1.70 ; \mathrm{CC}$ vs TT: $\mathrm{OR}=2.25,95 \% \mathrm{Cl}=1.85-2.73 ; \mathrm{CC}$ vs TC: $\mathrm{OR}=1.65,95 \% \mathrm{Cl}=1.36-2.01 ; \mathrm{CC}$ vs TT $+\mathrm{TC}: \mathrm{OR}=1.95,95 \% \mathrm{Cl}=1.63-2.34)$, but not in the North. In the subgroup analysis by source of controls, significantly increased risk was found in population-based studies $(\mathrm{C}$ vs T: $\mathrm{OR}=1.37,95 \% \mathrm{Cl}=1.21-1.56 ; \mathrm{CC}$ vs TT: $\mathrm{OR}=1.83,95 \% \mathrm{Cl}=$ 1.44-2.33; CC vs TC: $\mathrm{OR}=1.35,95 \% \mathrm{Cl}=1.07-1.72 ; \mathrm{CC}$ vs $\mathrm{TT}+\mathrm{TC}: \mathrm{OR}=1.58,95 \% \mathrm{Cl}=1.25-$ 2.01), but not in hospital-based studies.

\section{Sensitivity analysis and bias diagnosis}

To validate the credibility of the outcomes of this meta-analysis, a sensitivity analysis was performed by comparing results of random-effect and fixed-effect models. None of the results were substantially different with respect to the model type (Table 2). Hence, results of the sensitivity analysis suggest that the data in this meta-analysis are relatively stable and credible. Begg's funnel plot and the Egger test were employed to assess the publication bias of literatures. The shape of the funnel plots did not reveal obvious asymmetry (Figure 3 ). Then, the Egger test was used to provide statistical evidence of funnel plot symmetry. The Egger test indicated that there was no obvious publication bias under the allele model in the overall analyses $(t=0.84, P=0.412)$. 
Table 2. Primary results from the total and subgroup analyses.

\begin{tabular}{|c|c|c|c|c|c|c|}
\hline \multirow[t]{2}{*}{ Analysis model } & \multirow[t]{2}{*}{ Study groups } & \multirow[t]{2}{*}{$\mathrm{N}$} & \multirow{2}{*}{$\frac{\text { Random-effect model }}{\text { OR }(95 \% \mathrm{Cl})}$} & \multirow{2}{*}{$\frac{\text { Fixed-effect model }}{\text { OR }(95 \% \mathrm{Cl})}$} & \multicolumn{2}{|c|}{ Heterogeneity } \\
\hline & & & & & $x^{2}$ & $P$ \\
\hline \multirow[t]{5}{*}{ C vs T } & Total analysis & 19 & $1.34(1.16-1.54)$ & $1.29(1.21-1.37)$ & 81.17 & 0.000 \\
\hline & PB & 15 & $1.37(1.21-1.56)$ & $1.36(1.26-1.46)$ & 40.21 & 0.000 \\
\hline & $\mathrm{HB}$ & 4 & $1.21(0.75-1.95)$ & $1.04(0.91-1.20)$ & 30.93 & 0.000 \\
\hline & South China & 9 & $1.48(1.29-1.70)$ & $1.47(1.34-1.61)$ & 18.08 & 0.021 \\
\hline & North China & 10 & $1.22(0.98-1.51)$ & $1.13(0.96-1.24)$ & 47.54 & 0.000 \\
\hline \multirow[t]{5}{*}{ CC vs TT } & Total analysis & 19 & $1.73(1.30-2.30)$ & $1.65(1439-1.90)$ & 67.19 & 0.000 \\
\hline & PB & 15 & $1.83(1.44-2.33)$ & $1.85(1.58-2.16)$ & 29.94 & 0.008 \\
\hline & $\mathrm{HB}$ & 4 & $1.44(0.49-4.21)$ & $1.08(0.79-1.46)$ & 30.10 & 0.000 \\
\hline & South China & 9 & $2.23(1.83-2.70)$ & $2.25(1.85-2.73)$ & 7.65 & 0.468 \\
\hline & North China & 10 & $1.36(0.85-2.17)$ & $1.16(0.94-1.42)$ & 40.90 & 0.000 \\
\hline \multirow[t]{5}{*}{ CC vs TC } & Total analysis & 19 & $1.30(1.03-1.65)$ & $1.28(1.11-1.47)$ & 44.78 & 0.000 \\
\hline & PB & 15 & $1.35(1.07-1.72)$ & $1.36(1.16-1.60)$ & 28.68 & 0.012 \\
\hline & $\mathrm{HB}$ & 4 & $1.16(0.57-2.36)$ & $0.99(0.72-1.35)$ & 13.44 & 0.004 \\
\hline & South China & 9 & $1.67(1.30-2.13)$ & $1.65(1.36-2.01)$ & 11.80 & 0.160 \\
\hline & North China & 10 & $1.01(0.73-1.39)$ & $0.96(0.78-1.18)$ & 19.75 & 0.020 \\
\hline \multirow[t]{5}{*}{$\mathrm{CC}$ vs TT + TC } & Total analysis & 19 & $1.51(1.17-1.95)$ & $1.46(1.28-1.67)$ & 60.88 & 0.000 \\
\hline & PB & 15 & $1.58(1.25-2.01)$ & $1.60(1.38-1.85)$ & 33.08 & 0.003 \\
\hline & $\mathrm{HB}$ & 4 & $1.28(0.53-3.07)$ & $1.03(0.77-1.38)$ & 22.71 & 0.000 \\
\hline & South China & 9 & $1.94(1.57-2.41)$ & $1.95(1.63-2.34)$ & 10.52 & 0.230 \\
\hline & North China & 10 & $1.16(0.79-1.71)$ & $1.05(0.86-1.27)$ & 31.50 & 0.000 \\
\hline
\end{tabular}

PB = population-based, HB = hospital-based, South China includes Taiwan, Jiangsu, Sichuan, Zhejiang, Hubei, and Hunan; North China includes Beijing, Xinjiang, Shandong, and Shanxi.

Study
ID

Figure 2. Forest plot of CAD risk associated with APOA5 -1131T/C polymorphism (C vs T model). 


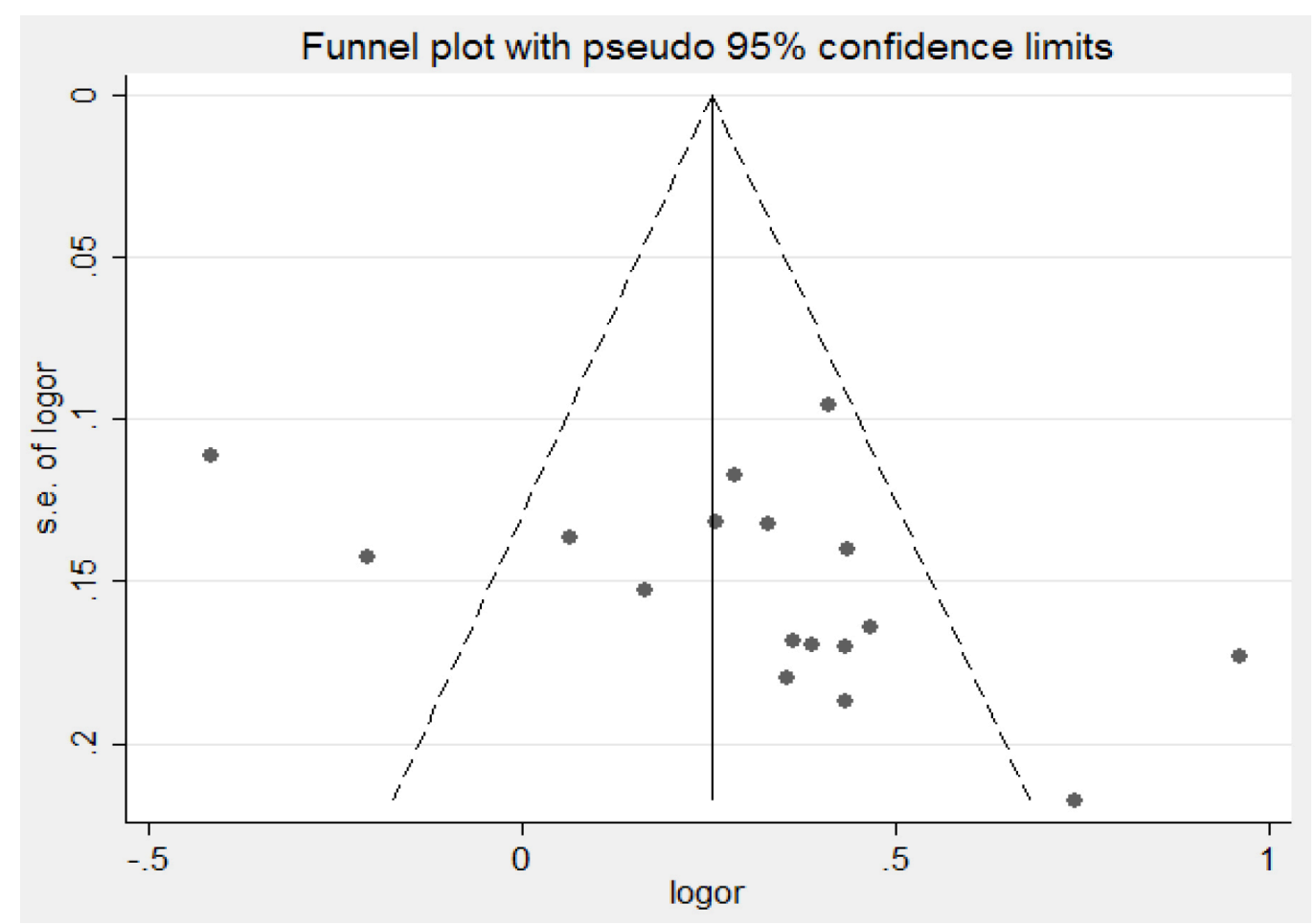

Figure 3. Begg's funnel plot of APOA5 -1131T/C polymorphism and CAD risk (C vs T model).

\section{DISCUSSION}

Despite several studies investigating APOA5 -1131T/C polymorphism and its association with $C A D$, researchers have not been able to derive definite conclusions. Until now, three metaanalyses have been published (Zhai et al., 2011; Zhang et al., 2011; Li et al., 2013b), of which, two were in Chinese population. Nevertheless, there are some drawbacks in these analyses, which could be improved on (Zhai et al., 2011; Li et al., 2013b). Therefore, we conducted an updated meta-analysis to derive a more precise estimation of APOA5 -1131T/C and susceptibility to CAD in Chinese population. Our meta-analysis involved 19 case-control studies with a total of 3983 CAD cases and 4358 controls. The overall results showed that a significantly elevated risk of CAD was associated with all APOA5 -1131T/C variants. Our results were consistent with the previously published meta-analysis in Chinese population (Zhai et al., 2011; Li et al., 2013b). However, these analyses did not investigate the modification of polymorphic genotypes by environmental risk factors and included a smaller number of studies on the Chinese than ours.

Further, we performed subgroup analyses stratified by geographical locations and source of controls to explore whether environmental factors can modulate this risk. We found that the APOA5 -1131T/C variants significantly increase the risk of CAD in South China and populationbased studies, but not in North China or hospital-based studies. One possible explanation is that the impact of this gene on CAD susceptibility might be ethnicity-based. However, we did not perform subgroup analysis based on nationality or other ethnicity due to lack of sufficient data. In addition, genetic backgrounds and the different living habits in South and North China could play 
an important role in susceptibility to CAD. In such cases, gene-gene and/or gene-environmental interactions need to be accounted as additional contributing factors. Moreover, hospital-based studies usually have some biases because such controls may just represent a sample of ill-defined reference population and may not be representative of the general population.

CAD is a multi-factorial and polygenic disorder, which is thought to be the result of interaction between an individual's genetic background and various environmental factors (Nora et al., 1980; Marenberg et al., 1994). The effect of any single gene might have a limited impact on CAD risk than has so far been anticipated. Therefore, some limitations of this study should be acknowledged. Firstly, our study evaluates only the influence of geographical location and source of control, whereas few investigators have reported the effects of this polymorphism under other environment factors such as smoking, alcohol intake, physical activities, and diets. Another potential limitation could be that our results are based on unadjusted estimates. Analyses that are more precise could be conducted if individual data were available, which would allow for the adjustment of covariates including age, gender, race, and other factors. Finally, heterogeneity can interfere with the interpretation of the results of a meta-analysis. Although we minimized this likelihood by performing a careful search of published studies and subgroup analyses, significant inter-study heterogeneity nevertheless existed in most of the comparison.

In conclusion, this meta-analysis suggests that the minor allele of the APOA5 -1131T/C polymorphism is a risk factor for CAD in Chinese, especially in the population from South China. Considering that CAD has multifactorial etiology, larger studies in selected populations with different environmental background or other risk factors are required to further evaluate the influence of genegene and gene-environment interactions on APOA5 -1131T/C polymorphism concerning CAD risk. Such studies may eventually lead to a better and comprehensive understanding of this association.

\section{Conflicts of interest}

The authors declare no conflict of interest.

\section{REFERENCES}

Bi N, Yan SK, Li GP, Yin ZN, et al. (2004). A single nucleotide polymorphismy $-1131 \mathrm{~T}>\mathrm{C}$ in the apolipoprotein A5 gene is associated with an increased risk of coronary artery disease and alters triglyceride metabolism in Chinese. Mol. Genet. Metab. 83: 280-286.

Bi N, Yan SK, Li GP, Yin ZN, et al. (2005). Polymorphisms in the apolipoprotein A5 gene and apolipoprotein C3 gene in patients with coronary artery disease. Chin. J. Cardiol. 33: 116-121.

Chen Y, Xu LP, Zhang JQ and Li R (2011). Study on the correlation of apolipoprotein A5 -1131T>C polymorphism and coronary heart disease. Chin. J. Convalescent. Med. 20: 487-489.

Cheng XQ, Yan SK, Song YH, Xiao XH, et al. (2007). Relationship between apolipoprotein AV gene -1131T>C polymorphism and type 2 diabetes mellitus with coronary artery disease in Han nationality. Mol. Cardiol. Chin. 7: 189-194.

Dai HY, Ye GH, Shi ZX and Zhao YC (2013). Relationship of APOA5 level and APOA5-1131T/C locus gene polymorphism to early-onset $\mathrm{CHD}$ and restenoses after percutaneous coronary intervention in Han population. Chin. General Pract. 16: 3821-3824.

Dalen JE, Alpert JS, Goldberg RJ and Weinstein RS (2014). The epidemic of the 20(th) century: coronary heart disease. Am. J. Med.127: 807-812.

Gaziano TA (2005). Cardiovascular disease in the developing world and its cost-effective management. Circulation 112: $3547-3553$.

Han TL (2011). A study on the haplotype of apolipoprotein A5/C3 gene cluster and correlation to the patients with coronary artery disease in Shandong coastal area. Master's thesis (Chinese), Shandong University, Shandong.

Han TL, Cai XL, Li M, Song DL, et al. (2010). Genovariation of apolipoprotein A5 in patients with coronary artery disease. Acta Acad. Med Qingdao Univ. 46: 512-514. 
Hokason JE and Austin MA (1996). Plasma triglyceride level is a risk factor for cardiovascular disease independent of highdensity lipoprotein cholesterol level: a meta-analysis of population-based prospective studies. J. Cardiovasc. Risk 3 : 213-219.

Hsu LA, Ko YL, Chang CJ, Hu CF, et al. (2006). Genetic variations of apolipoprotein A5 gene is associated with the risk of coronary artery disease among Chinese in Taiwan. Atherosclerosis 185: 143-149.

Jiang G, Wang D, Li W, Pan Y, et al. (2012). Coronary heart disease mortality in China: age, gender, and urban-rural gaps during epidemiological transition. Rev. Panam Salud Publica 31: 317-324.

Li XP, Zhao SP and Nie S (2007). Relationship between apolipoprotein A5 -I131T>C polymorphism and coronary heart disease. Chin. Circulation J. 22: 4-8.

Li X, Zhang Y, Wang M, Lv X, et al. (2013a). The prevalence and awareness of cardiometabolic risk factors in Southern Chinese population with coronary artery disease. Sci. World J. 2013: 416192.

Li YY, Wu XY, Xu J, Qian Y, et al. (2013b). Apo A5 -1131T/C, FgB -455G/A, -148C/T, and CETP TaqlB gene polymorphisms and coronary artery disease in the Chinese population: a meta-analysis of 15,055 subjects. Mol. Biol. Rep. 40: $1997-2014$.

Liu H, Zhang S, Lin J, Li H, et al. (2005). Association between DNA variant sites in the apolipoprotein A5 gene and coronary heart disease in Chinese. Metabolism 54: 568-572.

Marenberg ME, Risch N, Berkman LF, Floderus B, et al. (1994). Genetic susceptibility to death from coronary heart disease in a study of twins. N. Engl. J. Med. 330: 1041-1046.

Nora JJ, Lortscher RH, Spangler RD, Nora AH, et al. (1980). Genetic epidemiologic study of early-onset ischemic heart disease. Circulation 61: 503-508.

Pennacchio LA, Olivier M, Hubacek JA, Cohen JC, et al. (2001). An apolipoprotein influencing triglycerides in humans and mice revealed by comparative sequencing. Science 294: 169-173.

Qiu F (2007). The association of polymorphisms in apolipoproteina 5 gene with blood lipids and heart cerebrovascular disease. Master's thesis (Chinese), Southeast University, China.

Qiu F, Zhang K, Xu B, Gu GY, et al. (2007). The associated study of apolipoprotein A5 gene polymorphism with the degrees of coronary artery stenosis in coronary heart disease patients. Chin. J. Arterioscler. 15: 695-698.

Rosamond W, Flegal K, Friday G, Furie K, et al. (2007). Heart disease and stroke statistics - 2007 update: a report from the American Heart Association Statistics Committee and Stroke Statistics Subcommittee. Circulation 115: e69-e171.

Sayols-Baixeras S, Lluís-Ganella C, Lucas G and Elosua R (2014). Pathogenesis of coronary artery disease: focus on genetic risk factors and identification of genetic variants. Appl. Clin. Genet. 7: 15-32.

Szalai C, Keszei M, Duba J, Prohászka Z, et al. (2004). Polymorphism in the promoter region of the apolipoprotein A5 gene is associated with an increased susceptibility for coronary artery disease. Atherosclerosis 173: 109-114.

Tang YB (2006). Study on the association between apolipoprotein A5 genetic variations and susceptibility to coronary artery disease. Master's thesis (Chinese), Nanjing Medical University, Nanjing.

Tang YB, Sun P, Guo DP, Li XY, et al. (2005). Association between apolipoprotein A5 - 1131T \&gt; C polymorphism and susceptibility of coronary artery disease in Chinese. Zhonghua Yi Xue Yi Chuan Xue Za Zhi 22: 281-283.

van der Vliet HN, Sammels MG, Leegwater AC, Levels JH, et al. (2001). Apolipoprotein A-V: a novel apolipoprotein associated with an early phase of liver regeneration. J. Biol. Chem. 276: 44512-44520.

van Dijk KW, Rensen PC, Voshol PJ and Havekes LM (2004). The role and mode of action of apolipoproteins CIII and AV: Synergistic actors in triglyceride metabolism? Curr. Opin. Lipidol. 15: 239-246.

Wang CT (2004). SNP3,4,5 of the apolipoprotein A5 gene and their association with coronary artery disease in Chinese. Master's thesis (Chinese), Fujian Medical University, Fujian.

Xu L and He T (2008). Correlation of apolipoprotein A5 SNP3 gene single nucleotide polymorphism with coronary artery disease in Chinese. Shandong Med. J. 48: 1-3.

Yan SK, Cheng XQ, Song YH, Xiao XH, et al. (2005). Apolipoprotein A5 gene polymorphism -1131T->C: association with plasma lipids and type 2 diabetes mellitus with coronary heart disease in Chinese. Clin. Chem. Lab. Med. 43: 607-612

Yang F, Ng ZJ and Wang LS (2007). Association of APOA5 gene -1131T>C single nucleotide polymorphism with coronary heart disease among Chinese Han population. Shandong Med. J. 47: 1-3.

Yin SX (2006). A study on the genes single nucleotide polymorphism of apolipoprotein A5 and C3 in patients with coronary artery disease. Master's thesis (Chinese), Qingdao University, Qingdao.

Yu Y, Xue L, Zhao CY, Li JP, et al. (2007). Study on polymorphism in the apolipoprotein A5 gene in patients with premature coronary heart disease. J. Peking Univ. (Health Sciences) 39: 576-580.

Yuan S, Ma YT, Xie X, Yang YN, et al. (2011). Association of apolipoprotein A5 gene polymorphism with coronary heart disease in Uygur population of Xinjiang. Zhonghua Yi Xue Yi Chuan Xue Za Zhi 28: 73-77.

Zhai G, Li M and Zhu C (2011). APOA5 -1131T/C polymorphism is associated with coronary artery disease in a Chinese population: a meta-analysis. Clin. Chem. Lab. Med. 49: 535-539.

Zhang CJ (2009). The associations between Uygur, Han APOA5, APOB, CETP gene and lipids of coronary heart disease with diabetes. Doctoral thesis (Chinese), Xinjiang Medical University, Xinjiang. 
Zhang YQ (2007). Associations of apoA 5 polymorphisms, blood lipids and coronary heart disease in the Chinese. Master's thesis (Chinese), Zhejiang University, Zhejiang.

Zhang Z, Peng B, Gong RR, Gao LB, et al. (2011). Apolipoprotein A5 polymorphisms and risk of coronary artery disease: a meta-analysis. Biosci. Trends 5: 165-172.

Zhao L (2008). Association between apolipoprotein A5 -1131T>C polymorphism and coronary heart disease in xinjiang Uygur. Master's thesis (Chinese), Xinjiang Medical University, Xinjiang.

Zhu MA, Zhou YL, Ding Y and Mao DY (2007). Study on -1131T>C polymorphism of apolipoprotein A5 gene in patients with coronary artery disease. Chin. J. Gerontol. 73-75. 